\title{
Ethnopharmacology in the 21st century - grand challenges
}

\section{Michael Heinrich*}

Centre for Pharmacognosy and Phytotherapy, The School of Pharmacy, University of London, London, UK

*Correspondence: michael.heinrich@pharmacy.ac.uk

One can argue forever what precise percentage of the world's population use local and traditional medicines. These herbal (or mineral or fungal or occasionally animal) medical products form systems of knowledge and practice that have been transmitted over centuries and which continuously change. However, there can be no doubt that the majority of humans either rely on such products (often due to lack of other alternatives) or that herbal medicines are chosen consciously as an alternative to mainstream medicine. In some cases this knowledge is documented in an extensive historical written body of scholarly and applied writings. Traditions like "Traditional" Chinese, Ayurvedic, Unani, Jamu, Kampo, Iranian, Aztec or various forms of European and Arabic medicine are well known examples. In other regions we rely on the efforts of researchers past and present to document such knowledge and to critically analyze the data. It is now a commonplace that such "traditional knowledge" is disappearing fast, but in fact it is simply changing under the pressures of a globalizing world.

Frontiers in Ethnopharmacology is a worldwide, open access platform for bioscientific, clinical and sociocultural research on medicinal and food plants as well as other natural substances used throughout the world. This multidisciplinary approach is an essential basis for the use of these resources in tomorrow's medicines. Neglected diseases, including such common ones as vector-borne ones, diarrhea or tuberculosis are still commonly treated with herbal medicines. There is a growing use of plants to manage or treat AIDS/HIV or other emerging or fast spreading diseases (like viral respiratory diseases. Novel treatment strategies are needed for all diseases and in recent years herbal medicines from such traditions have received particular attention in the management (prevention or treatment) of chronic diseases like diabetes, chronic CNS disorders and many forms of cancer. Lastly, there are major gaps in our knowledge both about the general safety and the interaction potential of these medicines.

A core challenge in pharmacology is the scientific study of the complex products derived from such traditions. Extracts obtained from plants, fungi or animals pose some unique challenges: they are multicomponent mixtures of active, partially active and inactive substances and the activity is often not on a single target. Because of this complexity, extracts often vary and thus the pharmacological effects cannot always be reproduced. And they offer unique opportunities. The structural diversity and chemical complexity of natural products is unbeaten. In areas like anti-cancer and anti-parasitic medications as well as in pain management, natural products form the core of human's treatment options: "Artemisinin, triptolide, celastrol, capsaicin, and curcumin are "poster children" for the power and promise of turning traditional medicines into modern drugs. However, their stories highlight the ongoing interdisciplinary research efforts that continue to be necessary to realize the pharmaceutical potential of traditional therapeutics" (Corson and Crews, 2007). While a lot of research is going on in the field of ethnopharmacology, much of this work is either poorly accessible or it lacks a critical and state-of-the art approach. This is one of the grand challenges in ethnopharmacology.

Pharmacology has been influenced tremendously by its links with studies on "natural products" (Malone, 1983; Heinrich, 2010). For example, in the 19th century the first systematic studies on the pharmacological effects of curare were conducted by the French physiologist/pharmacologist Claude Bernard (1813-1878). This is a classical example of an in vivo approach which - in combination with ex vivo approaches dominated pharmacology until the advent of screening oriented and later more mechanistic in vitro experiments (Heinrich, 2010). Of course, the serendipitous discovery of the penicillins (Alexander Flemming,
$1881-1955)$ in 1928 is the classical example of how successful science has been in refining and downscaling the experimental setups and in focusing these on a single target. A further core step were the development of in vitro methods to screen for novel anticancer agents. This line of research started in the late 1950's at the US NCI/NIH and resulted in the famous discoveries of the vinca-alkaloids, taxol, camptothecin and podophyllum-derivatives as medicinal agents as well as a much more detailed understanding of their mechanism of action (in two cases associated with Monroe Wall (1916-2002), Mansukhlal C. Wani (* 1925) and co-workers, Sneader, 1985).

Since the 1970's in vitro methods became more and more important. A wide range of targets were explored (e.g., antimicrobial effects, Ieven et al., 1979; anti-viral effects, Van den Berghe et al., 1978, 1986; monamine oxidase inhibitors, Suzuki et al., 1981, angiotensin inhibitors, Elbl and Wagner, 1991; anti-inflammatory and anti-allergic effects, Wagner, 1989). Microplate-based routine methods and other approaches to miniaturize sample size marked a new step in these developments, also allowing for an increase in the overall throughput of samples (Suzuki et al., 1981). Another approach was developed from the 1980's onwards by combining thin layer chromatography techniques first with overlay techniques to detect anti-microbiological activity and then the use of microbial or chemical spraying reagents in order to detect biological activities. Examples include the use of Bacillus subtilis (Hamburger and Cordell, 1987) or the use of DPPH as a model for anti-oxidant effects. Bioautography had originally been used to study, for example, the strength and purity of penicillins, but was now explored in the systematic ethnopharmacological study of traditional and local medicinal plants. The search for anti-protozoal agents was also pushed forward by the use of in vitro techniques, which started in the early 1980's (Wright and Phillipson, 1990). Since the 1980 's a wide range of in vitro studies were 
published (Houghton et al., 2007), which, however, were often not carried forward towards a more mechanistic biomolecular and pharmacological understanding of these products or their clinical development. Recent developments have again taken these challenges further and we now see an interest in such clinical and more molecular biology oriented strategies (Corson and Crews, 2007; Graz et al., 2010).

All these are examples of ethnopharmacological approaches where researchers very successfully combined approaches from the chemical, biological and pharmacological sciences. Thus, the further development of these techniques using state-of-the art technology is another core challenge. As in other areas of drug discovery, translating this into products for use in biomedicine has been far less successful, a problem which may well be overcome using these novel approaches.

Another core aspect of the challenge relates to the complexity of plants and other natural resources and the extracts or products derived from them. In the 19th century the initial discoveries of natural products started the search for novel medicines (Heinrich, 2010). These discoveries were only possible thanks to, what we would now call, interdisciplinary research efforts linking biology and chemistry with pharmacology (like Claude Bernard's work). However, especially during 1970's and 1980's studies identifying novel compounds from plants or fungi without a link to ethnopharmacology are much more numerous. Frontiers in Ethnopharmacology encourages truly transdisciplinary approaches.

A wide range of innovations in phytochemical analysis allowed an ever faster analysis and isolation of natural products and their identification/structure elucidation. However, the complexity of mixtures as they are found in extracts from natural sources continues to be a challenge in ethnopharmacology/natural product research. TLC and later HPLC fingerprinting offered opportunities to characterize extracts and in the last two decades hyphenated techniques allowed scientists to identify many of the metabolites in an extract in a relatively short period of time. It has recently been argued that metabolomics offers unique opportunities to understand such complex mixtures, and this is most certainly one step further in understanding such mixtures (Verpoorte et al., 2005, Heinrich, 2008; Schripsema, 2010).

Therefore, where are the opportunities and challenges for the future? We need studies at all levels from the critical study of traditional and local knowledge systems (Etkin, 1988, Heinrich et al., 2009), to the screening of such plants in primary assays, the detailed mechanistic study of an extract's or a natural compound's pharmacology and toxicology to the clinical evaluation of their effectiveness (Corson and Crews, 2007; cf. Graz et al., 2010) or studies on the public health implications of using (or not using) such medicines. All this forms the basis for an evidence-based approach to these "natural medicines". In general terms the safety of such products is considered to be more and more a central area of research. In recent years some therapeutic areas have proven to be of particular relevance, including diabetes, chronic and acute inflammatory conditions and anti-infective agents. By definition, this research has to be transdisciplinary and we trust that authors will submit work which addresses this complexity using state-of-the art methodologies. Molecular biology, pharmacogenomics, genomics, proteonomics and related disciplines now offer such approaches (Efferth et al., 2008). When used in the context of the study of medicinal and food plants and their pharmacological activities, such approaches offer exciting new insights which Frontiers in Ethnopharmacology will incorporate.

Of course, research in this field not only needs to comply with the ethical requirements of the sciences, but also with national and international laws relating to the protection and equitable and sustainable use of biodiversity and the rights of the original keepers of this knowledge (Secretariat of the Convention on Biological Diversity, 2001). This is linked to the sustainable use of such resource and environmental protection of the world's biocultural diversity and the development of novel product for human and veterinary use are all aspects of Frontiers in Ethnopharmacology's strategic focus.

Frontier in Ethnopharmacology will hopefully develop fast and will constantly incorporate changes brought about by a changing, continuouslyglobalizing and more and more web-based scientific world.

\section{ACKNOWLEDGMENTS}

I am grateful to Th. Efferth (Mainz) and many colleagues and co-authors for useful discussions about the strategic opportunities in Ethnopharmacology

\section{REFERENCES}

Corson, T.W., and Crews, C.M. (2007). Molecular understanding and modern application of traditional medicines: triumphs and trials. Cell 130, 769-774.

Efferth, T., Kahl, S., Paulus, K., Adams, M., Rauh, R., Boechzelt, H., Hao, X., Kaina, B., and Bauer, R. (2008). Phytochemistry and pharmacogenomics of natural products derived from traditional chinese medicine and chinese materia medica with activity against tumor cells. Mol. Cancer Ther. 7, 152-161.

Elbl, G., and Wagner, H. (1991). A new method for the in vitro screening of inhibitors of angiotensin converting enzyme ACE), using the chromophore- and fluorophore-labelled substrate, dansyltriglycine. Planta Med. 57, 137-141.

Etkin, N., (1988). Ethnopharmacology, biobehavioral approaches in the anthropological study of indigenous medicines. Ann. Rev. Anthropol. 17, 23-42.

Graz, B., Falquet, J., and Elisabetsky, E. (2010). Ethnopharmacology, sustainable development and cooperation: the importance of gathering clinical data during field surveys. J. Ethnopharmacol. 130. doi: 10.1016/j.jep.2010.04.044 [Epub ahead of print].

Hamburger, M. O., and Cordell, G. A. (1987). A direct bioautographic TLC assay for compounds possessing antibacterial activity. J. Nat. Prod. 50, 19-22.

Heinrich, M. (2008). Ethnopharmacy and natural product research - multidisciplinary opportunities for research in the metabolomic age. Phytochem. Lett. 1, 1-5.

Heinrich, M. (2010). "Ethnopharmacology and drug development," in Comprehensive Natural Products II Chemistry and Biology Vol. 3, eds L. Mander and H.-W. Lui (Elsevier: Oxford), 351-381.

Heinrich, M., Edwards, S., Moerman, D. E., and Leonti, M. (2009).Ethnopharmacological field studies: a critical assessment of their conceptual basis and methods. J. Ethnopharmacol. 124, 1-17.

Houghton, P. J., Howes, M.-J., Lee, C.C., and Steventon, G. (2007). Uses and abuses of in vitro tests in ethnopharmacology: visualizing an elephant. J. Ethnopharmacol. 110, 391-400.

Ieven, M., van den Berghe, D.A., Mertens, F., Vlietinck, A. and Lammens, E. (1979). Screening of higher plants for biological activities. I. Antimicrobial activity. Planta Med. 36, 311-321.

Malone, M.H. (1983). The pharmacological evaluation of natural products - general and specific approaches to screening ethnopharmaceuticals. J. Ethnopharmacol. 8, 127-147.

Schripsema, J. (2010). Application of NMR in plant metabolomics: techniques, problems and prospects. Phytochem. Anal. 21, 14-21.

Secretariat of the Convention on Biological Diversity. (2001). Handbook of the Convention on Biological Diversity. London: Earthscan Publ.

Sneader,W. (1985). Drug Prototypes and their Exploitation. Chichester: J. Wiley and Sons.

Suzuki, O., Katsumata, Y., Oya, M., Chari, V. M., Vermes, B., Wagner, H., and Hostettmann, K. (1981). Inhibition 
of type-A and type-B monamine oxidases by naturally occurring xanthones. Planta Med. 42, 17-21.

Van den Berghe, D. A., Leven, M., Mertens, F., and Vlietinck, A. J. (1978). Screening of higher plants for biological activity, II. Antiviral activity. Lloydia 41, 463-471.

Van den Berghe, D. A., Vlietinck, A. J., and Van Hoof, L. (1986). Plant products as potential antiviral agents. Bull. Inst. Pasteur. 84, 101-147.

Verpoorte, R., Choi, Y. H., and Kim, H. K. (2005). Ethnopharmacology and systems biology: a perfect holistic match. J. Ethnopharmacol. 100, $53-56$.

Wagner, H. (1989). Search for new plant constituents with potential antiphlogistic and antiallergic activity. Planta Med. 55, 235-241.

Wright, C.W., and Phillipson, J. D. (1990). Natural products and the development of selective antiprotozoal drugs. Phytother. Res. 4, 127-129.

Received: 03 June 2010; accepted: 07 June 2010; published online: 28 June 2010.
Citation: Heinrich M (2010) Ethnopharmacology in the 21st century - grand challenges. Front. Pharmacol. 1:8. doi: 10.3389/fphar.2010.00008

This articlewassubmitted to Frontiersin Ethnopharmacology, a specialty of Frontiers in Pharmacology

Copyright (C) 2010 Heinrich. This is an open-access article subject to an exclusive license agreement between the authors and the Frontiers Research Foundation, which permits unrestricted use, distribution, and reproduction in any medium, provided the original authors and source are credited. 